\title{
Herausforderungen und Chancen für die Arbeit der Akademie für Ethik in der Medizin angesichts der COVID-19-Pandemie
}

\author{
Alfred Simon · Christin Zang
}

Online publiziert: 13. Mai 2020

(C) Springer-Verlag GmbH Deutschland, ein Teil von Springer Nature 2020

Die gegenwärtige COVID-19-Pandemie stellt unser Gesundheitssystem vor große Herausforderungen und wirft eine Reihe grundlegender Fragen ethischer Natur auf: Ist es verhältnismäßig, zum Schutz von Risikogruppen sowie aus Sorge vor einer möglichen Überlastung des Gesundheitssystems, flächendeckend freiheitsbeschränkende Maßnahmen zu verhängen? Dürfen bereits geplante Untersuchungen und Operationen abgesagt bzw. verschoben werden, um medizinische Ressourcen für die Behandlung von COVID-19-Patient*innen vorzuhalten? Welche lebensbedrohlich Erkrankten sollen im Falle nicht ausreichender intensivmedizinischer Ressourcen priorisiert werden? Dürfen Alten- und Pflegeheime ihren Bewohner*innen verbieten, Besuch von Angehörigen zu empfangen? Was ist für eine Bewohner*in einer solchen Einrichtung schlimmer: die Gefahr der Ansteckung mit einer für sie möglicherweise tödlich verlaufenden Erkrankung oder die totale soziale Isolation - und wer darf das (für sie) entscheiden? Das Besondere an diesen Fragen ist, dass sie Entscheidungen in sehr kurzer Zeit verlangen, für die es häufig keine angemessene Evidenz- und mitunter auch keine ausreichende Rechtsgrundlage gibt und deren mittel- und langfristigen Folgen zum Zeitpunkt der Entscheidung oft nicht absehbar sind.

Die Akademie für Ethik in der Medizin (AEM) versteht sich als medizinethische Fachgesellschaft. Wie kann sie den Diskurs über ethische Fragen im Zusammenhang mit COVID-19 fördern? Was kann sie tun, um Ärzt*innen, Pflegende und andere im Gesundheitsbereich Tätige bei ihren schwierigen Entscheidungen zu unterstützen? Worin sieht sie ihre Rolle in der aktuellen Krisensituation?

Prof. Dr. A. Simon $(\bowtie) \cdot$ C. Zang, M.A.

Akademie für Ethik in der Medizin e. V., Humboldtallee 36, 37073 Göttingen, Deutschland

E-Mail: simon@aem-online.de 
Ein zentrales Ziel der AEM ist es, den wissenschaftlichen wie auch öffentlichen Diskurs über medizinethische Fragen zu fördern. Diesem Selbstverständnis entsprechend hat die AEM eine Sonderseite zu COVID-19 auf ihrer Homepage eingerichtet, auf der u. a. Materialien und Empfehlungen zu ethischen Fragen der Patientenversorgung, zu forschungsethischer Literatur sowie zum Thema E-Learning im Rahmen der Medizinethiklehre für Medizinstudierende zu finden sind. ${ }^{1}$ Die Informationen auf der Seite stammen von Mitgliedern sowie einschlägigen Arbeitsgruppen; die Aktualisierung der Seite liegt in den Händen der Geschäftsstelle.

An die AEM wurde schon früh die wichtige Frage herangetragen, welche Rolle Klinische Ethik und Ethikberatung im Rahmen der COVID-19-Pandemie spielen kann und soll. Um diese Frage breit und bedarfsorientiert zu diskutieren, bietet die AEM - inspiriert durch eine Initiative Schweizer Kolleg*innen - seit Mitte März regelmäßige Online-Meetings an, an denen bis zu 150 Personen aus dem gesamten Bundesgebiet sowie vereinzelt auch aus Österreich, Tschechien und der Schweiz teilnehmen. Neben dem Austausch von Erfahrungen und Informationen, gaben diese Meetings auch Anstoß für konkrete Projekte: So wurde im Anschluss an das erste Meeting von der AEM ein Online-Forum ${ }^{2}$ eingerichtet, in dem sich klinische Ethiker*innen über ethische Fragen und Themen im Zusammenhang mit COVID-19 austauschen können. Darüber hinaus wurde vom Vorstand der AEM unter Beteiligung weiterer Mitglieder ein Diskussionspapier zu „Möglichkeiten und Grenzen von Ethikberatung im Rahmen der COVID-19-Pandemie " in deutscher ${ }^{3}$ und englischer ${ }^{4}$ Sprache erarbeitet. Und von einer Ad-hoc-Arbeitsgruppe wurde eine Handreichung zu „Psychische Belastungen von Gesundheitspersonal im Umgang mit moralischen Konflikten"5 erstellt. Ein weiteres Papier, das sich mit den spezifisch pflegeethischen Fragen im Zusammenhang mit COVID-19 beschäftigt, wird aktuell (Stand: April 2020) von Vertreter*innen der beiden Arbeitsgruppen „Pflege und Ethik" in der AEM vorbereitet.

Neben den von ihr selbst initiierten Projekten unterstïtzt die AEM den Aufruf für eine Nationale Taskforce „COVID-19-Evidenz" ${ }^{\text {“6 }}$, der eine professionelle Priorisierung, Koordinierung und Kommunikation der Forschung zu nicht-pharmakologischen Interventionen fordert. Außerdem ist die AEM beteiligt am „Kompetenznetz Public Health COVID-19“7 und engagiert sich dort insbesondere in einer Arbeits-

\footnotetext{
1 https://www.aem-online.de/index.php?id=162 (zugegriffen: 24.04.2020).

2 https://forum.aem-online.de (zugegriffen: 24.04.2020).

3 https://www.aem-online.de/fileadmin/user_upload/AEM_Ethikberatung_im_Rahmen_von_COVID19_2020-03-31.pdf (zugegriffen: 24.04.2020).

4 https://www.aem-online.de/fileadmin/user_upload/AEM_Recommendations_Role_of_COVID-19_ Pandemic_2020-03-31.pdf (zugegriffen: 24.04.2020).

5 https://www.aem-online.de/fileadmin/user_upload/Handreichung_psychischer_Belastung_15_4_final. pdf (zugegriffen: 24.04.2020).

6 https://www.aem-online.de/fileadmin/user_upload/Aufruf_Taskforce_COVID-19-Evidenz-5_4.pdf (zugegriffen: 24.04.2020).

7 https://www.public-health-covid19.de/ (zugegriffen: 24.04.2020).
} 
gruppe, die sich mit den ethischen Fragestellungen, welche speziell Public Health betreffen, beschäftigt und hierzu eine Handreichung erarbeitet hat ${ }^{8}$.

Ferner hat der Vorstand der AEM zusammen mit sechs weiteren Fachgesellschaften klinisch-ethische Empfehlungen zu „Entscheidungen über die Zuteilung von Ressourcen in der Notfall- und der Intensivmedizin im Kontext der COVID19-Pandemie“"9 veröffentlicht mit dem Ziel, den klinischen Praktiker*innen eine ethische Orientierung bei ethisch schwierigen und belastenden Entscheidungen zu geben. Viele der darin enthaltenen Empfehlungen sind unkontrovers (z. B. die Warnung vor sozialer Diskriminierung), einige sind aber auch Gegenstand kontroverser fachlicher und öffentlicher Debatten, die bis in den Vorstand der AEM hineinreichen: So konnte z.B. die Empfehlung, dass eine Priorisierung nach dem Kriterium der klinischen Erfolgsaussicht im Falle objektiv nicht ausreichender intensivmedizinischer Ressourcen nicht nur „ex ante“, sondern auch „ex post“ erfolgen solle, nicht von allen Vorstandsmitgliedern persönlich mitgetragen werden. Trotz der inhaltlichen Differenz war sich der Vorstand aber einig, die Empfehlungen, an deren Erarbeitung mehrere Mitglieder der AEM federführend mitgewirkt haben, mit Mehrheitsbeschluss zu unterstützen. Zugleich bot sich ein Vorstandsmitglied an, das ethische Dilemma, das mit solchen Priorisierungsentscheidungen verbunden ist, in einem ethischen Hintergrundkommentar ${ }^{10} \mathrm{zu}$ beleuchten.

Es ist bereits angeklungen, dass aufgrund der Dynamik der COVID-19-Pandemie eine enge Vernetzung und ein schneller Austausch über Fachdisziplinen, Professionen und Ländergrenzen hinweg zur Beantwortung dringender ethischer Fragen notwendig sind. Aus dem Bestreben heraus, frühzeitig Diskurse zu ermöglichen, hat sich die AEM u.a. entschieden, Diskussionspapiere als vorläufige Versionen $\mathrm{zu}$ veröffentlichen und mithilfe des Feedbacks dann prozessbegleitend zu optimieren. Zudem hat die AEM mit dem Online-Forum und den Online-Meetings neue Kommunikationswege eingerichtet, um räumliche Distanz zu überwinden.

Die AEM sieht sich aber auch Herausforderungen in ihren eigenen regulären Tätigkeiten gegenüber. So kann die Jahrestagung 2020 nicht in gewohnter Form stattfinden. Wann und in welcher Form die Mitgliederversammlung durchgeführt wird, steht zum aktuellen Zeitpunkt (April 2020) noch nicht fest. Kleinere Veranstaltungen, wie die Schreibwerkstatt „Publizieren in der Medizinethik“ im Juni, oder Arbeitsgruppensitzungen finden in Form von Online-Konferenzen statt. Und auch der Vorstand selbst hat sich in den vergangenen Wochen regelmäßig über OnlineKonferenzen ausgetauscht.

Obwohl 2020 eine Vielzahl an Fort- und Weiterbildungsangeboten und insbesondere Kurse zu „Ethikberatung im Gesundheitswesen“ ausgesetzt werden müssen, ist die Zahl der Anträge auf Zertifizierung im Vergleich zum Vorjahr weiter angestiegen. Außerdem zeichnet sich ab, dass die Zahl der Neumitgliedschaften angesichts

\footnotetext{
8 https://aem-online.de/fileadmin/user_upload/Policy_Brief_Pandemic_Ethics_GeneralF.pdf (zugegriffen: 24.04.2020).

9 https://aem-online.de/fileadmin/user_upload/200416-divi-covid-19-ethik-empfehlung-version-2.pdf (zugegriffen: 24.04.2020).

10 https://www.aem-online.de/fileadmin/user_upload/Ralf-Stoecker-Ethischer-Hintergrundkommentarzur-Stellungnahme-der-Fachverbaende-und-AEM-1.pdf (zugegriffen: 24.04.2020).
} 
der neuen Herausforderungen und der gestärkten Vernetzung einen Anstieg erfahren könnte.

Die COVID-19-Pandemie mag diverse Umstellungen erzwungen haben, durch das Betreten neuer Wege bietet sich nun jedoch mehr denn je die Chance, mit der Digitalisierung und Globalisierung mitzuwachsen sowie die dazugewonnen Erkenntnisse und neuen Arbeitsformen für zukünftige Diskurse fruchtbar zu machen.

Interessenkonflikt A. Simon ist Leiter und C. Zang wissenschaftliche Mitarbeiterin der Geschäftsstelle der AEM. 